\title{
Polysèmes
}

Revue d'études intertextuelles et intermédiales

$7 \mid 2005$

Les figures de la violence

\section{Destins de violence}

Françoise Fonteneau

\section{OpenEdition}

\section{Journals}

Édition électronique

URL : http://journals.openedition.org/polysemes/1642

DOI : $10.4000 /$ polysemes. 1642

ISSN : 2496-4212

Éditeur

SAIT

Édition imprimée

Date de publication : 1 janvier 2005

Pagination : 27-41

ISSN : 0999-4203

\section{Référence électronique}

Françoise Fonteneau, "Destins de violence », Polysèmes [En ligne], 7| 2005, mis en ligne le 01 janvier 2005, consulté le 19 avril 2019. URL : http://journals.openedition.org/polysemes/1642 ; DOI :

10.4000/polysemes.1642

Ce document a été généré automatiquement le 19 avril 2019.

Polysèmes 


\title{
Destins de violence
}

\author{
Françoise Fonteneau
}

1 Le lien entre la violence et l'irreprésentable, la présence de la violence dans le discours comme événement dans le texte, sont des questions qui se découpent derrière les «Figures de la violence ». Mais qui fait violence? Qui fait violence à qui ? Quel est le destin de cette violence inhérente à un discours, quel en est le destinataire ? De quel sujet vient-elle?

\section{Violence et pulsion}

2 «Violence » est un terme peu usité en psychanalyse. Pourtant c'est ce terme que Freud choisit d'employer lorsqu'il répond à A. Einstein en 1932 sur le thème «Pourquoi la guerre ? $»^{1}$ (Warum Krieg ?). Il choisit d'employer délibérément le mot « violence » (Gewalt) à la place du mot « pouvoir» (Macht) et du mot «droit » (Recht). Pour répondre à la question de Einstein, Freud propose donc un changement de vocabulaire : «Oserais-je remplacer le mot de pouvoir par celui plus cru et plus dur de violence?». Droit et violence semblent aujourd'hui antinomiques mais les conflits entre les hommes ont été autrefois fondamentalement tranchés par le recours à la violence. Le droit a commencé par le droit $\mathrm{du}$ plus fort, quasiment par la force musculaire, puis par celui des meilleures armes. Puis on a vu la violence d'un groupe s'opposer à la violence d'un seul. «Le droit - écrit Freud - est la force d'une communauté, mais il s'agit toujours d'une violence, toujours prête à se retourner contre tout individu qui s'oppose à elle, elle opère avec les mêmes moyens, elle poursuit les mêmes buts». Freud va jusqu'à parler «d'actes de violence légaux ». On a même vu des guerres se terminer en faisant croire que le pouvoir avait apporté la paix à des conflits locaux. Pensons à l'Empire romain et à la pax romana mais aussi à des conflits européens plus récents (Bosnie, Kosovo...).

3 Freud montre aussi dans sa lettre qu'il y a deux facteurs de cohésion d'une communauté, d'une société : les liens affectifs entre les membres, mais aussi la violence vers l'ennemi extérieur ou intérieur. Comment aller plus loin dans l'explication freudienne ? C'est que l'homme est un être de pulsion (Trieb) et que cette pulsion est double, bifide : tantôt eros, la pulsion de vie, Lebenstrieb, l'amour, amour de soi aussi, autoconservation. La pulsion 
amoureuse peut devenir pulsion d'appropriation, et peut tourner à l'agressivité dans la conquête de l'objet aimé. Tantôt thanatos, la pulsion de mort, Todestrieb, parfois exercée vers l'extérieur, parfois vers le sujet lui-même. Freud, s'engageant sur le mot « violence » en est venu tout naturellement à faire une mise au point sur la pulsion, double, bifide. Il faut dire qu'il ne rate jamais une occasion pour le faire. Que ce soient des occasions cliniques ou des occasions sociales et politiques (comme dans le Malaise dans la civilisation, ou Les Considérations sur la guerre), voire littéraires, lorsqu'il se saisit du personnage de la Rebecca d'Ibsen ou bien de Lady Macbeth de Shakespeare qu'il prend comme exemples de " caractères de ceux qui échouent à cause du succès " ${ }^{2}$. Il entend dénoncer l'hypocrisie qu'il y a à croire l'homme bon, cultivé, soumis ou engagé dans une éthique, alors que celle-ci n'est, la plupart du temps, qu'un simple emplâtre, une simple thérapeuthique. La façon dont la violence, l'agressivité sont traitées dans notre société, dit Freud, c'est-à-dire non prises en compte, fait que la violence ne peut que rendre malade et l'agresseur et l'agressé. Le Surmoi collectif civilisé (le Kulturüberich) va jouer seulement sur le sentiment de culpabilité : «C'est une hérésie d'avoir voulu expliquer la naissance de la conscience morale par le retournement de l'agression vers l'intérieur ». C'est la position de Freud annonçant le Surmoi comme un des trois « despotes » : le monde extérieur, le Surmoi et le ça ${ }^{3}$. L'être vivant préserve pour ainsi dire sa propre vie en détruisant celle d'autrui, mais une partie de la pulsion reste active à l'intérieur de lui. On le voit dans toutes sortes de phénomènes normaux et pathologiques.

\section{Violence du mot, violence de la lettre}

Venons-en à l'individu, au sujet souffrant à qui nous avons affaire dans la psychanalyse. Dans ce cadre nous travaillons d'une part dans la particularité et d'autre part, avec et par la parole avec des mots, sur des discours. Si l'analyste avait pour tâche de détecter les " figures de la violence », ce serait au un par un, ce serait faire surgir pour le patient, via son histoire, ce qu'est la violence pour lui, comment il la cultive peut-être, comment il s'y emploie vis-à-vis de lui-même ou des autres.

5 Le patient commence souvent par construire son histoire, son « roman familial ». Il va trouver là des responsables de la violence qu'il a pu subir, des auteurs de violence, souvent sous forme de prononceurs de paroles agressives et déterminantes. Il trouve quelqu'un à haïr, quelqu'un à aimer, quelqu'un auprès de qui quémander, quelqu'un qui a voulu pour lui, quelqu'un à qui il a soumis son désir, désir sur lequel il a sûrement cédé. Le poids des mots, ou des non-dits, des silences se feront peser, soupeser, longtemps peutêtre, avant que le sujet n'apprenne à faire arrêt à sa souffrance, au malaise, n'apprenne à stopper la jouissance morbide qu'il pouvait entretenir, à lâcher cette souffrance. De ce poids des mots, des silences, comme violence faite au sujet, je donnerai quelques exemples empruntés tantôt à la psychanalyse, tantôt à la littérature, ou au cinéma.

\section{Le Président Schreber, « martyr de l'inconscient »}

6 Mon premier exemple sera la violence des "paroles imposées» dans l'hallucination verbale chez le psychotique. J'évoquerai ici le Président Schreber dont le cas clinique nous est connu par ses propres Mémoires ${ }^{4}$, et par les travaux que Freud et Lacan ont effectués sur ce cas ${ }^{5}$. Schreber part d'un état de fait qu'il nomme «contrainte au jeu continu de la pensée », terme qu'il dit tenir des voix intérieures elles-mêmes et qui repose 
sur le fait « qu'on force quelqu'un à penser sans relâche ». Il existe pour lui, en dehors de la parole humaine ordinaire, une sorte de "parler de nerfs" dont en règle générale l'homme normal n'est pas conscient. Dans ce «parler de nerfs", les mots sont récités en silence - comme dans une oraison mentale-mais les organes spécifiques de la parole n'interviennent pas. Dans les conditions normales ce « parler de nerfs » ne dépend que de la volonté de l'être humain, mais dans son cas de malade, Schreber explique que «ses nerfs viennent à être mobilisés de l'extérieur, continûment et sans répit ». Il s'agit là selon lui d'une « contrainte au jeu continu de la pensée » que, en bon juriste qu'il est, il décrète être " une atteinte portée aux droits les plus fondamentaux de l'homme ». On voit très bien la violence extrême de ce «jeu forcé de la pensée ». Schreber n'est jamais dans le silence, silence qu'il associe à l'être mental sain. Lacan évoque cet état de fait chez le psychotique en employant cette expression: "martyr de l'inconscient». En somme pourrait-on dire, «le psychotique est un martyre de l'inconscient, en donnant au sens de martyr son sens qui est d'être témoin $»^{6}$. À quoi correspond le martyr du psychotique ? Ce martyre correspond à une absence totale de silence pour Schreber. Il associe le silence maîtrisé à la maîtrise des facultés mentales, et l'arrivée des moments de maladie, de délires, il les associe à la présence d'un silence qui ne dépend plus de lui, qui se remplit sans cesse, comme un trou, d'un trop plein de paroles, de mots. Autant de signifiants qui extérieurement ou intérieurement lui sont imposés par le «jeu forcé de la pensée ». Ce deuxième silence devient l'entonnoir dans lequel s'engouffrent les pensées inconscientes non-maîtrisées. Lorsque Lacan parle de la pulsion invocante - celle des voix, de la voix comme de celle qui est la plus proche de l'expérience de l'inconscient, il nous dit que c'est avec cette idée en tête qu'il nous faut examiner les phénomènes d'hallucination verbale, des voix. Le sujet qui entend des voix, qui n'est, comme Schreber, jamais au silence, jamais au repos, perçoit l'objet de sa voix comme présent ${ }^{7}$. Le psychotique est un martyr, parce qu'il est un témoin qui est hors d'état de restaurer le sens de ce dont il témoigne et de le partager dans le discours des autres.

7 Chez l'homme normal ou le névrosé, le signifiant finit par surgir - ne serait-ce que sous forme de symptôme - sur fond de silence. Il ne peut pas surgir de la même manière chez le psychotique. Schreber aspire à la paix, au silence, à l'absence de voix, mais c'est aussi le silence qui le coince dans le délire, ouvre la porte aux hallucinations, laisse couler ce qu'avec jouissance il ne cesse de ne pas retenir8. L'une des violences faite à Schreber est qu'il n'est jamais dans l'absence de sens. Un signifiant est là, toujours prêt à le surprendre, mais qui jamais ne se dévoile, dit Lacan. Il parle d'un «signifiant inconscient » qui, dans la psychose, semble extérieur au sujet, mais auquel le sujet reste attaché par une fixation érotique?.

\section{Meurtre d'un bookmaker chinois (The Killing of a Chinese Bookie) film de 1976-1978 de John Cassavetes}

8 Mon deuxième exemple sera emprunté au cinéma, au personnage de Cosmo Vitelli dans Meurtre d'un bookmaker chinois, film de John Cassavetes, 1976-1978.

9 Dans Les Considérations sur la guerre, Freud reprend à son compte une expression de Balzac, "Tuer son mandarin». "Tuer son mandarin » pour Balzac, était l'idée qu'un homme pourrait, par un simple acte de volonté, tuer à Pékin un vieux mandarin dont le décès ne manquerait pas de lui apporter un grand avantage. Tuer pour un bénéfice, désirer la mort de quelqu'un et, par la «toute puissance des idées » - expression du patient de Freud 
l'homme aux rats - tuer en restant caché, en faisant silence, est l'idée que Freud prend pour exemple pour mettre en évidence les désirs de mort qui agissent en silence et la logique du névrosé de la « toute puissance de la pensée ».

En apparence, rien de tout cela chez le héros du film de Cassavetes. Cosmo Vitelli, patron du Crazy Horse de Los Angeles, a contracté au jeu d'énormes dettes, auprès de la mafia. Il ne peut les rembourser. On lui propose, en échange de ses dettes, d'assassiner un bookmaker chinois, fort dangereusement entouré. Sa vie est en jeu, la mafia le tuera s'il ne rembourse pas. On lui propose donc de se dédouaner, d'effacer sa dette en commettant un meurtre. Il s'agit pour lui de tuer le vieux bookmaker chinois, non pas de loin, mais de près, en acte, et non pas en pensée.

11 Nous sommes dans une problématique du style « la bourse ou la vie? », où l'on sait qu'on ne gagne jamais vraiment mais que l'on perd toujours quelque chose. Pourtant ce que fait semblant de croire Vitelli, c'est qu'il y a une monnaie d'échange possible. Comme certains sujets s'acharnent à faire des équivalences, à croire en des équivalences possibles, Vitelli fait semblant de croire que sa vie, menacée par la mafia, pourrait équivaloir à celle d'un autre, sa mort à celle d'un autre, l'argent à une mort. Substitution, déplacement, condensation, qui équivalent à ceux des formations de l'inconscient, le mènent à effectuer sa mission : un meurtre dont il ne sortira pas indemne. La violence se retourne contre lui. Il réalisera la vanité de son acte, la tromperie des autres, mais surtout la tromperie de lui-même à lui-même. À la fin, Cosmo, blessé, touché, proche de la mort, prend conscience qu'on ne peut rien donner en échange de sa vie - serait-ce la mort d'un vieux bookmaker chinois. Il laisse le clown de sa revue dire à sa place : « I can't give you anything but love ». "Rien à donner hors l'amour ", telle pourrait être sa devise finale. Or, l'amour dira Lacan, c'est justement « donner ce qu'on n'a pas »10.

\section{Bartleby le scribe : contingence et nécessité, où le manque de partenaire soutenant la lettre fait surgir la pulsion de mort}

Mon troisième exemple évoquera Bartleby le scribe ${ }^{11}$, personnage de Melville.

Comment Bartleby le scribe de Melville renonce-t-il à la lettre, mais en meurt-il dès qu'on ne la lui demande plus ? Comment le passage d'une contingence à une nécessité pourra-til conduire à la mort ? Nous voyons dans ce texte en quoi la formule de Bartleby : «I would prefer not to ", " je préférerais ne pas » finit par équivaloir à un vœu de mort. Lorsqu'il ne lui est plus permis de prononcer cette formule, lorsque plus personne n'adresse de demande à Bartleby - comme l'a fait un certain temps l'homme de loi dans le bureau demande à laquelle il pouvait répondre par cette phrase énigmatique, "I would prefer not to ", il tombe dans la nécessité de la mort, de la non-vie.

Pour Deleuze ${ }^{12}$, la formule «I would prefer not to... » s'anéantit elle-même. Bartleby ne refuse pas, il n'affirme pas non plus un préférable, il récuse un «non-préféré ». S'il disait oui, s'il disait non, il n'y survivrait pas. Pour Deleuze la formule « fait souffler un vent de folie ", " un souffle psychotique passe dans le langage ", même si Bartleby, lui, ne possède pas de procédé comme un Brisset ou un Wolfson. Sa formule annonce le silence, le retrait derrière le paravent, le silence final, la mort. Il est sans références, sans particularité : «I am not a particular ». Deleuze montre qu'on est obligé de tenir compte des anomalies de l'homme de loi, face à ce vent de folie. Mais il me semble qu'on peut aller plus loin encore dans le partenariat entre Bartleby et l'homme de loi. Dans quelle position avait voulu se 
placer ce dernier? Est-ce celle du père ? De l'homme de loi philanthrope, protecteur? Le "I would prefer not to" destitue le père de sa parole. Il ne reste plus que la fraternité possible à l'égard de Bartleby. Et pourtant, pas plus que le bon père bienveillant, le grand frère protecteur ne parviendra à sauver Bartleby. Bartleby réclamait un peu de confiance. L'homme de loi a tenté de la lui accorder. Mais, englué dans la charité, dans la philanthropie, la bonne morale, tous les masques de la fonction paternelle, ce qu'il nomme sa "friandise ", (a sweet morsel), il ne lui fait confiance que peu de temps. Coupant court à toute possibilité de contingence, assénant la nécessité comme celle d'un ordre vital, il cessera de poser à Bartleby les questions qui pouvaient lui permettre d'exister - dans la contingence pure. Quand ce temps-là de la demande cessera, il sera dans le silence et mourra. La nécessité face au futur n'est pas seulement face à un événement - bataille navale ou pas demain, écrire ou ne pas écrire pour le scribe - mais face à l'alternative être ou ne pas être, exister ou ne pas exister. Bartleby n'est ni dans le refus, ni dans la dénégation pure, il est au-delà. Au delà d'Hamlet, au-delà de la morale. Il ne cède pas sur son désir, si faible, si non-défini, si inattendu soit ce dernier. Mais il a besoin pour vivre d'un partenaire qui soutiendra ce minimum vital du désir en puissance, contingent ; un partenaire qui résiste à l'inattendu, l'insu que Bartleby lui impose, hors des circuits de la morale, du Bien, du Mal.

La fraternité ne suffit pas à soutenir le maigre désir vide de Bartleby. Et si le désir de l'autre n'est plus manifesté, si la demande cède, il s'écroule. Son apparente résistance à la demande, au désir de l'autre qui semble le soutenir sur le fil du rasoir, ne fonctionne que le temps de cette demande. Le désir vide, la contingence pure s'écroule sans le soutien de l'autre. Alors, l'amour ou la mort?

\section{Le « voeu de mort »}

Dans son séminaire sur "Les formations de l'inconscient $»^{13}$, Lacan fait s'équivaloir la demande de mort et la mort de la demande, en particulier chez le névrosé obsessionnel. Il en fait une demande primordiale, "le voeu de mort", au même titre que la demande d'amour. Elle s'adresse à l'Autre susceptible de satisfaire ou de frustrer le sujet, d'accéder à son désir ou non. Mais elle s'adresse à ce grand Autre avec ambivalence. Dès que la demande est adressée à l'Autre, dès qu'elle s'ébauche, elle s'éteint. Le désir s'émousse, se meurt, en tous cas il est réprimé sous forme d'une dénégation (Verneinung). Cependant, même dénié ce désir sera capable d'entraîner la culpabilité. Le fait que l'Autre soit le lieu de la demande implique la mort de la demande. Du fait de cette demande déniée, le désir est ressenti comme interdit. C'est pourquoi une certaine frustration de la demande peut s'accompagner d'une disparition du désir et on voit pointer là une douloureuse culpabilité, sous forme de " avoir cédé sur son désir ».

Bien sûr, s'agissant de la demande de mort, à l'horizon, il y a le commandement « tu ne tueras point! ». L'homme aux rats, un patient de Freud, expliquait à ce dernier que ses premiers contenus obsessionnels lui arrivaient sous forme de commandements. Par exemple, au moment où son amie le quitte pour aller soigner une vieille tante : «Va! et assassine la vieille femme!». Puis plus tard «Tue-toi! pour te punir d'avoir eu de tels désirs ». Si le grand Autre est celui qui peut interdire le désir, le sujet va chercher sa permission, non pas auprès du petit autre auquel il s'adresse, mais vers un grand Autre qui n'existe pas. 

l'objet qui représente le sein maternel. La demande est tout de suite dédoublée : demande du sein et demande d'amour absolue. Ce premier rapport, rapport de dépendance, est menacé déjà par la perte d'amour. L'horizon de la demande, dans le cas de l'obsessionnel, est suspendu à la formation précoce de la demande de mort. Pour illustrer sa thèse, Lacan évoque le silence fréquent de l'obsessionnel comme demande de mort de la demande. Cette demande de mort primordiale, énigmatique, qui marque les tous premiers rapports de l'obsessionnel avec l'Autre, Lacan la rapproche de la question des rapports du désir et de la nature, évoquant là la démarche de la Naturphilosophie.

Nous pourrions poser la question : cette demande de mort, est-ce pour devancer la perte, l'abandon, la disparition, le deuil, diverses formes de violence redoutées - que le sujet s'y trouve? Elle semble venir d'une nature retournée contre elle-même. Elle peut empêcher toute articulation de la demande du sujet obsessionnel, faire obstacle à son discours. Il est dans une impossibilité de parler. Le dédoublement de la demande - du fait qu'elle est toujours demande absolue d'amour, place le sujet dans un balancement, une ambivalence. Le sujet parlant ne peut pas atteindre l'Autre sans s'atteindre lui-même, dit Lacan, si bien que la demande de mort est la mort de la demande. Face aux diverses formes de violence évoquées, est-il possible de distinguer entre désir d'analyste et désir d'écrivain?

\section{Désir d'écrivain, désir d'analyste}

20 À la violence du silence, à la violence du mot, à la violence du signifiant sur le sujet, psychanalystes et écrivains sont confrontés. Je terminerai en dissociant désir d'écrivains et désir de psychanalystes.

21 L'auteur, l'écrivain, présente souvent merveilleusement la névrose, voire la psychose. Il les met en scène. Il déploie les mécanismes d'échec, de répétition, les façons dont un personnage cède sur son désir avec une jouissance morbide. Je trouve à cet égard Henry James particulièrement doué pour peindre ces situations. Beaucoup de ses personnages féminins, par exemple dans Une vie à Londres ou dans Les Dépouilles de Poynton, excellent dans cet art de nier le désir, de viser l'échec de l'amour, de transformer le possible en impossible au point que le renversement en fabrique une certaine grandeur et noblesse, un certain héroïsme du personnage. On est au-delà du sacrifice pour un autre, un petit autre, on prétend se réaliser dans le malheur. Le désir est irrecevable ou indicible, les passions sont tues ou avortées. On est dans le silence, le secret, puis dans le renoncement, la résignation, le malheur. La violence à l'égard de soi-même est exacerbée mais aussi sublimée. La jouissance en est superbement mise en évidence.

L'écrivain décrit, met en scène. Il dit les mécanismes de répétition, d'échec, d'une violence à soi-même ou à l'autre, les déchaînements de cette violence. S'il dénonce, écrit les difficultés du sujet à dire, à écrire l'innommable, l'irreprésentable, l'indicible, est-ce là que s'arrête son objectif ? Ou bien attend-il aussi de son texte un effet de violence, ou un effet cathartique? De quelle nature est son désir quant à son impact sur le lecteur?

L'analyste lui, conduit une cure pour un sujet. Il ne laisse pas seulement se déployer les mécanismes de répétition, les échecs, les fantasmes, les diverses figures de la violence. Il fait acte. S'il laisse une place vide, parfois silencieuse, où peuvent se déployer, prendre adresse, ces discours violents, il attend le moment propice pour agir, visant le surgissement du sujet et pas seulement son bien-être, ce "service des Biens » comme

Polysèmes, 7 | 2005 
disait Lacan. Freud mettait déjà en garde les analystes de vouloir le Bien de leurs patients. Lacan, lui, dénonce les idéaux de la psychanalyse : l'amour médecin, le démasquage, la non-dépendance. Il les dénonce, s'ils ne sont pas visés dans le sens du désir du sujet. Et l'éthique de la psychanalyse mesure - comme toute éthique - le rapport de l'action au désir. Avez-vous agi conformément au désir qui vous habite? dit Lacan, ou bien vous êtes-vous trahi? Quel est le prix à payer pour l'accès au désir? La jouissance, la livre de chair. L'analyste vise le moment où le sujet ne cédera pas sur son désir, enfin détaché de celui de l'Autre, le grand, pour être enfin réalisé avec l'autre, le petit, quittant aliénation dans une séparation. L'éthique de l'analyste se situe donc par rapport à ce désir.

Si l'analyste avait pour tâche de détecter les figures de la violence, et il n'aurait, il faut le dire, la plupart du temps que l'embarras du choix des matériaux, agressivité, sadisme, pulsion de mort, ce serait dans son travail au un par un. Ce serait faire surgir pour un patient dans sa particularité ce qu'est pour lui la violence (pulsion de mort) comment il la cultive, s'y emploie à l'égard de lui-même ou des autres.

Un pas aussi est visé pour le sujet et peut se faire dans le sens d'une réalisation que la violence des mots ne lui est pas propre, mais propre à tout usage de la langue. Cette violence on peut la situer dans l'incapacité des mots à dire, à cerner la Chose. Dans la violence de la lettre qui fait trou, motus, meurtre. Les mots sont impuissants; jamais le signifiant ne rejoindra le signifié. C'est cet aspect de la violence des mots qu'on peut avec Jean-Claude Milner nommer la « castration linguistique ».

Accepter cela, faire avec, accepter qu'on ne peut que mi-dire la vérité, est le pas que le patient pourra faire dans l'analyse. Il ne s'agit pas, bien sûr, de céder au silence de la pulsion de mort sa place ${ }^{14}$. Pas plus que le poète ne fait le deuil du mot, même si le « mot faillit », l'analyste ni l'analysant ne céderont sur le dire ${ }^{15}$. De toutes façons, l'inconscient, lui, parle.

Le désir de l'analyste est que le sujet fasse lui-même arrêt à la jouissance morbide, qu'il parvienne à lâcher la souffrance, à ne plus se faire violence. Faire dessiner à un sujet les figures de violence qui lui sont propres, forgées dans son histoire, lui en faire saisir le fonctionnement, son fonctionnement, lui permettre de s'en extraire, voire de s'en servir - comme il peut finir par se servir de son symptôme - est ce que vise l'analyste, un sujet dont le destin de violence n'a plus rien à voir avec une fatalité ${ }^{16}:$ "L'éthique de la psychanalyse s'articule d'une orientation du repérage de l'homme par rapport au réel $»^{17}$. Ce réel, tissé de violence, impossible à dire, à écrire, et «qui ne cesse pas de ne pas s'écrire $»^{18}$.

\section{BIBLIOGRAPHIE}

Cassavetes, John. The Killing of a Chinese Bookie [Meurtre d'un bookmaker chinois], film de 1976-1978.

Deleuze, Gilles. Critique et clinique. Paris : Éditions de Minuit, 1993.

Fonteneau, Françoise. L'Éthique du silence. Paris : Éditions du Seuil, 1999. 
Freud, Sigmund. « Pourquoi la guerre? ». In Résultats Idées Problèmes II. Paris : PUF, 1985.

Freud, Sigmund. «Quelques types de caractère : Ceux qui échouent à cause du succès ». In L'Inquiétante étrangeté. Paris : Gallimard, 1985.

Freud, Sigmund. XXXI ${ }^{e}$ conférence « La décomposition de la personnalité psychique ». In Nouvelles conférences d'introduction à la psychanalyse. Paris : Gallimard, 1984.

Freud, Sigmund. « Remarques psychanalytiques sur l'autobiographie d'un cas de paranoïa ». In Cinq psychanalyses. Paris : PUF, 1954.

Lacan, Jacques. Le Séminaire, Livre III, « Les psychoses ». Paris : Éditions du Seuil, 1981.

Lacan, Jacques. Le Séminaire, Livre V, «Les formations de l'inconscient ». Paris : Éditions du Seuil, 1998.

Lacan, Jacques. Le Séminaire, livre VII, «L'éthique de la psychanalyse ». Paris : Éditions du Seuil, 1986.

Lacan, Jacques. Le Séminaire, Livre VIII, « Le transfert ». Paris : Éditions du Seuil, 1991.

Lacan, Jacques. Le Séminaire, Livre XI, « Les quatre concepts fondamentaux de la psychanalyse ». Paris : Éditions du Seuil, 1973.

Lacan, Jacques. Le Séminaire, Livre XX, « Encore ». Paris : Éditions du Seuil, 1975.

Melville, Herman. Bartleby le scribe. Paris : Gallimard, 1995 [Éd. anglaise : Billy Budd, Sailor and other Stories, London, Penguin books, 1985].

Schreber, D.P. Mémoires d'un névropathe. Paris : Éditions du Seuil, 1985.

\section{NOTES}

1. Sigmund Freud, « Pourquoi la guerre? » in Résultats, Idées, Problèmes II, Paris, PUF, 1985. Warum Krieg? in Studienausgabe Band IV, 275.

2. Sigmund Freud, «Quelques types de caractères... » II, « ceux qui échouent à cause du succès ", in L'Inquiétante étrangeté, Paris, Gallimard, 1985.

3. Sigmund Freud, « XXI ${ }^{\mathrm{e}}$ conférence. La décomposition de la personnalité psychique» in Nouvelles conférences d'introduction à la psychanalyse, Paris, Gallimard, 1984, 107.

4. D. P. Schreber, Mémoires d'un névropathe, Paris, Éditions du Seuil, 1985.

5. Sigmund Freud, «Remarques psychanalytiques sur l'autobiographie d'un cas de paranoïa », in Cinq psychanalyses, Paris, PUF, 1954.

6. Jacques Lacan, Le Séminaire, Livre III «Les psychoses », Paris, Éditions du Seuil, 1981, 149.

7. «L'hallucination n'est pas un faux perceptum mais un percipiens dévié », Jacques Lacan, Le Séminaire, Livre XI, «Les quatre concepts fondamentaux de la psychanalyse », Paris, Éditions du Seuil, 1973, 232.

8. Schreber et ses rapports au silence et au bruit et à la voix, ne sont pas sans lien avec le rapport à la voix du Père, à l'Autre de la loi.

9. Jacques Lacan, Le Séminaire livre III, op. cit., 156-157.

10. Jacques Lacan, Le Séminaire Livre VIII « le transfert », Paris, Éditions du Seuil, 1991, 46 et 415 : « ...et on ne peut aimer qu'à se faire comme n'ayant pas, même si on l'a. L'amour comme réponse implique le domaine du non-savoir.» cf. aussi «Signification du phallus», in Écrits, Paris, Éditions du Seuil, 1966.

11. Herman Melville, Bartleby le scribe, Paris, Gallimard, 1995, [éd. anglaise : Billy Budd, Sailor and other Stories, London, Penguin books, 1985]. 
12. Gilles Deleuze, Critique et clinique, Paris, Minuit, 1993.

13. Jacques Lacan, Le Séminaire, Livre V, « Les formations de l'inconscient », Paris, Seuil, 1998.

14. Même si, comme le dit Lacan « la mort, en tant qu'elle est la base, l'opération du saint-esprit par laquelle le signifiant existe » (Séminaire IV, op. cit., 48 cf. le schéma des parallèles signifiantsignifié « qui fonctionne sur le fond d'une certaine expérience de la mort »).

15. Françoise Fonteneau, L'Éthique du silence, Paris, Seuil, 1999.

16. Sur l'idée de "destin» et la psychanalyse, cf. par exemple Jacques Lacan, Séminaire VIII, chap. 22, 322.

17. Jacques Lacan, Séminaire VII, «L'éthique de la psychanalyse », Paris, Éditions du Seuil, 1986, 21.

18. Jacques Lacan, Séminaire XX, «Encore », Paris, Éditions du Seuil, 1975, 132. Cf. aussi « Nomina non sunt consequentia rerum » in Ornicar? $n^{\circ} 16$ : «Le réel est l'impossible seulement à écrire, soit ne cesse pas de ne pas s'écrire. Le réel, c'est le possible en attendant qu'il s'écrive. »

INDEX

oeuvrecitee Bartleby the Scrivener, Killing of a Chinese Bookie (The) 